\title{
Linx
}

Revue des linguistes de l'université Paris X Nanterre

$82 \mid 2021$

Entre vieillissement et innovation : le changement linguistique

\section{Le rôle du cinétisme discursif et sémantique des mots dans le vieillissement et/ou la régénération des valeurs sociales épaisses : travail, innovation, démocratie, enseignant, réclame, publicité}

The role of discursive and semantic kinetics of words in the aging and/or regeneration of thick social values: work, innovation, democracy, teacher, advertising

Olga Galatanu

\section{OpenEdition}

Journals

Édition électronique

URL : https://journals.openedition.org/linx/8023

DOI : 10.4000/linx.8023

ISSN : $2118-9692$

Éditeur

Presses universitaires de Paris Nanterre

Référence électronique

Olga Galatanu, « Le rôle du cinétisme discursif et sémantique des mots dans le vieillissement et/ou la régénération des valeurs sociales épaisses : travail, innovation, démocratie, enseignant, réclame, publicité », Linx [En ligne], 82 | 2021, mis en ligne le 15 juillet 2021, consulté le 20 juillet 2021. URL : http://journals.openedition.org/linx/8023 ; DOI : https://doi.org/10.4000/linx.8023

Ce document a été généré automatiquement le 20 juillet 2021.

Département de Sciences du langage, Université Paris Ouest 


\section{Le rôle du cinétisme discursif et sémantique des mots dans le} vieillissement et/ou la régénération des valeurs sociales épaisses : travail, innovation, démocratie, enseignant, réclame, publicité

The role of discursive and semantic kinetics of words in the aging and/or regeneration of thick social values: work, innovation, democracy, teacher, advertising

Olga Galatanu

\section{Introduction : objectifs et hypothèses de départ}

1 L'objectif premier de cet article relève d'un projet dans le domaine de la théorie sémantique: proposer et argumenter une approche complémentaire de celles qui étudient le changement sémantique et les phénomènes qui lui sont associés, le vieillissement et l'innovation dans le domaine du sens linguistique. Nous nous proposons d'inscrire ainsi à la croisée de deux interfaces, synchronie-diachronie et signification lexicale-sens discursif, un phénomène que nous avons appelé le cinétisme discursif et sémantique du sens linguistique ${ }^{1}$.

2 En définissant le concept de cinétisme du sens linguistique, nous avons avancé l'hypothèse que ce phénomène est inhérent à l'activité langagière, qu'il représente le principe explicatif même $d u$ fonctionnement du sens linguistique, qu'il est donc présent dans toutes les occurrences de parole et pour toutes les catégories sémantiques des mots mobilisés dans ces occurrences². 
3 Cette première hypothèse situe les variations du sens discursif dans un rapport de réciprocité avec la signification des entités linguistiques. D'une part, la signification d'un mot autorise le déploiement de ses potentialités, de nature argumentative et axiologiquement orientées, mais aussi leur transgression dans certaines limites de la zone sémantique ${ }^{3}$; d'autre part, le sens discursif confirme, voire renforce même ces potentialités (1), ou au contraire, les affaiblit (2), ou les déconstruit pour les reconstruire, allant jusqu'à proposer une modification sémantique (3) ${ }^{4}$.

(1) C'est une vraie démocratie, le pouvoir doit appartenir au peuple.

(2) Ce n'est pas une véritable démocratie, le pouvoir n'appartient pas au peuple.

(3) C'est une démocratie, mais elle est efficace et le pouvoir appartient vraiment au peuple.

4 L'exemple 3 propose une reconstruction sémantique: pour comprendre le sens discursif du mot démocratie, et le considérer comme sémantiquement acceptable, il faut accepter aussi que dans sa signification (tout au moins le temps de cette occurrence discursive), il y a un élément appartenant au domaine modal pragmatique avec une orientation négative, l'inefficacité, qui n'est pas activé dans l'énoncé puisque l'enchaînement argumentatif transgressif mobilise la séquence «mais elle est efficace ", de même qu'un élément modal déontique concernant le pouvoir qui n'appartient pas vraiment au peuple.

Un deuxième objectif est de bâtir un pont conceptuel et opérationnel entre ce principe explicatif du fonctionnement du sens linguistique et les fonctions du discours dans le monde social ${ }^{5}$.

6 Expliquer et argumenter l'hypothèse que le lien entre les évolutions du monde social et le langage mis en discours, est biunivoque, ou réciproque ${ }^{6}$ revient à dire que le discours, par le sens discursif qu'il génère et par les significations lexicales que celui-ci propose, (a) (re)construit le monde social, les systèmes de valeurs et les identités, les faits institutionnels, et (b) peut être à l'origine du phénomène de vieillissement, ou au contraire, de régénération de mots qui désignent ces faits institutionnels, ces systèmes de valeurs et ces identités (Galatanu, 2000a,b).

7 Le corollaire de ces deux hypothèses de départ concerne directement la thématique de ce numéro. On peut avancer, dans la perspective théorique proposée, une explication du changement sémantique, dans une perspective sémasiologique, fondée sur le principe du cinétisme discursif et sémantique et sur les rapports bi-orientés qu'il entretient avec ce que l'on peut appeler le cinétisme culturel. C'est, par exemple, le cas du mot innovation devenu la dénomination d'une véritable valeur sociale complexe positive ${ }^{7}$.

8 On peut ainsi proposer une explication sémantique, dans une perspective sémasiologique, du vieillissement de certains mots, comme le mot réclame, vieilli et pratiquement "évincé " par le mot publicité en français de France. Enfin, on peut proposer une explication du lien entre le changement du sens d'une entité lexicale (cf. Blank, 2000) et le changement de désignation (Koch, 2000), comme une conséquence du changement du sens de cette entité lexicale et de son vieillissement.

9 L'article se propose d'illustrer nos propositions dans une zone sémantique particulièrement sensible pour l'interface <signification lexicale - sens discursif $>$, celle des valeurs sociales complexes, épaisses. 


\section{Vieillissement et régénération du lexique dans la zone des valeurs sociales complexes}

10 Les significations des mots qui désignent les valeurs sociales complexes sont porteuses de plusieurs valeurs modales et donc de phénomènes intrinsèques de sur-modalisation (Galatanu, 2000a, 2018). Nous avons appelé ces processus de modalisation inhérents à la signification lexicale modalisations internes et le résultat de ce processus valeurs modales épaisses ${ }^{8}$. Les entités lexicales porteuses de valeurs modales épaisses, comme démocratie, innovation, travail ont, dans cette perspective théorique, le statut de formes modales et désignent des valeurs sociales complexes. L'expression est inspirée du concept de Putnam de "valeur éthique épaisse» (Putnam, 2005), concept que nous avons élargi et redéfini, afin de rendre compte de la complexité des modalisations internes à la signification des mots (Galatanu, 2018).

11 La zone sémantique des valeurs modales épaisses est une zone "sensible ", au sens de «sensitive » en anglais, " délicat, susceptible de produire des débats, voire des conflits, des affects", mais aussi au sens de "susceptible de réagir au contact, à de faibles variations". Tout d'abord, les valeurs modales épaisses concernées sont orientées axiologiquement et en tant que telles proposées souvent par les discours comme universellement partagées. Mais, en même temps, elles sont mouvantes dans leur substantialité complexe, parfois paradoxale. Ensuite, les significations de ces mots représentent une grande variété de configurations sociales: faits sociaux, voire institutionnels (démocratie, républicain, citoyen, etc.), activités humaines et pratiques sociales (collaborer, travailler, travail, publicité, innovation), identités professionnelles ou autres (enseignant, paysan, politicien, francophone, islamiste, patient, client, etc.). Les valeurs modales, parmi lesquelles les valeurs axiologiques, modalisent, à l'intérieur même de la signification des mots, ces faits sociaux, activités, identités collectives, etc. ${ }^{9}$.

Bréal avait formulé avec clarté son objectif, s'intéressant aux « lois qui président à la transformation des sens, aux choix des expressions nouvelles, à la naissance et à la mort des locutions » (Bréal, $1897:$ 133) et, plus précisément à celles qu'il appelle « les lois intellectuelles du langage ».

Depuis et à travers différentes démarches visant à modéliser le changement sémantique (Sweetser, 1990; François et alii, 2000), ces faits, envisagés comme des causes ou des déclencheurs du changement sémantique, concernent essentiellement les rapports entre le changement du monde référentiel et celui des représentations de ce monde, telles qu'elles sont construites et proposées par la langue, en l'occurrence par les expressions lexicales, et par les discours qui les mobilisent.

14 En fait, il s'agit d'étudier, pour les prédicats nominaux, le changement d'un lien dénominatif (Kleiber, 1984, 2003). Par exemple, le parcours de signifiance du mot travail, dans les études, parfois polémiques ${ }^{10}$, de son étymologie, est envisagé comme un passage, probablement par un processus de métaphorisation, de la désignation (par un signifiant qui a subi, lui-même des transformations conformes à des lois phonétiques) d'un objet à valeurs praxéologiques et culturelles négatives à la désignation d'une activité qui garderait des traces de ces valeurs négatives.

15 D'autre part, on peut dire pour résumer d'une manière un peu rapide, que la démarche qui part de l'analyse des changements du monde social et humain et des évolutions de 
la connaissance du monde naturel, cherche à rendre compte de l'innovation lexicale et terminologique, congruente à ces changements et à ces évolutions.

C'est souvent le cas des discours institutionnels (Oger et alii, 2003; Longhi \& Sarfati, 2014) qui (re)définissent des faits institutionnels, voire même, des institutions, suivant les évolutions sociales.

Dans la perspective théorique que nous proposons, la Sémantique des Possibles Argumentatifs - la SPA -, nous défendons l'idée que le cinétisme discursif et sémantique du sens linguistique de ces mots modifie les valeurs, identités et faits institutionnels et participe ainsi à la (re)construction du monde social, conformément à l'hypothèse 2 avancée dans l'introduction de cet article. Cette approche rejoint l'approche de Searle de la construction du monde (social) par des actes déclaratifs qui instaurent (cf. Searle, 1969, 1975, 2010) soit une nouvelle institution sociale, soit des «faits institutionnels », définis par leurs statuts-fonctions ${ }^{11}$, dans le cadre d'institutions humaines déjà existantes.

Pour notre part, il s'agit d'étudier le cinétisme de mots qui désignent des faits institutionnels déjà existants, leur conférant des statuts-fonctions régénérés ou carrément reconstruits.

19 Le second volet de l'explication que nous proposons avance l'idée que le processus de modification des valeurs sociales complexes portées par les mots qui désignent ces nouveaux faits institutionnels (par exemple, leur flexion de polarité) peut générer un phénomène de vieillissement des mots concernés allant jusqu'à leur remplacement par un autre mot.

20 Nous allons argumenter l'idée que le principe explicatif du fonctionnement du sens linguistique, qui permet d'émettre et de conforter cette hypothèse est celui du cinétisme discursif et sémantique. Nous allons argumenter également une hypothèse locale sur le vieillissement du mot réclame et sur son remplacement par le mot publicité.

\section{Le cinétisme discursif et sémantique, principe explicatif du fonctionnement du sens linguistique}

21 Notre cadre théorique, la SPA, est une théorie du potentiel (descriptif, argumentatif et axiologiquement orienté) des significations des mots de produire du sens discursif. Elle est également une théorie du potentiel du sens discursif de confirmer et de conforter les significations linguistiques, en particulier des mots, ou au contraire de les régénérer, voire même de les déconstruire pour les reconstruire, ne serait-ce que le temps de l'occurrence de parole. Autrement dit, la SPA est une sémantique du cinétisme permanent du sens linguistique, susceptible toutefois de préserver de façon relativement durable, un noyau de propriétés essentielles des significations apprises et partagées à un moment donnée par une communauté linguistique (Galatanu, 2018) ${ }^{12}$. Nous allons rappeler quelques éléments qui nous ont permis de définir le concept de cinétisme discursif et sémantique. 


\subsection{Point de vue observationnel du langage et de son fonctionnement sémantique ${ }^{13}$} du monde conceptualisé et proposé par les significations apprises et partagées par la communauté linguistique et culturelle, mais susceptibles d'être régénérées ou reconstruites par les actes de parole, par les discours et l'inter-discours. Ce second point de vue observationnel du langage fonde et justifie un postulat empirique sur le statut argumentatif du sens discursif et son corollaire sur la configuration argumentative de la signification. Il fonde également l'hypothèse centrale de la SPA sur la (re)construction de la signification avec chaque occurrence de sens discursif. L'hypothèse explicite cet aspect du point de vue observationnel du langage, appréhendé comme outil de communication, id est de négociation et d'argumentation du sens discursif.

\subsection{Le modèle SPA du fonctionnement du sens linguistique}

L'objectif premier de la SPA est d'expliquer et de décrire la signification linguistique, notamment lexicale, comme "un processus toujours recommencé » de "révision de l'univers « référentiel » que le discours propose dans et par des actes langagiers ». Le modèle que les hypothèses internes de la SPA permettent de construire doit être susceptible d'expliquer ce processus.

\subsubsection{Hypothèse 1}

La SPA fait l'hypothèse de l'existence de plusieurs «strates » de la signification lexicale, qui n'est pas homogène au niveau du statut théorique des éléments signifiants qui la forment. Son corollaire est que cette hétérogénéité habilite les mécanismes qui soustendent le cinétisme discursif et sémantique. Les éléments signifiants qui configurent la signification et les sens discursifs d'un mot « sont des associations argumentatives de représentations sémantiques portées par d'autres mots du lexique d'une langue, associations ayant des degrés de stabilité et de durabilité différents » (Galatanu, 2018 : 176), et également des degrés de pertinence différents.

\subsubsection{Hypothèse 2}

La deuxième hypothèse pose la strate la plus stable, celle du noyau $(\mathrm{N})$ : une configuration vectorielle d'enchaînements argumentatifs de propriétés essentielles ${ }^{14}$ à la reconnaissance d'un mot. Elle avance ainsi l'existence d'une syntaxe argumentative organisant des liens spécifiques entre les mots de la langue d'appartenance, mots qui 
désignent les propriétés essentielles du mot concerné par l'analyse $\mathrm{e}^{15}$. On peut dire qu'en SPA, le noyau de la signification d'un mot représente en soi une ontologie sémantique de la connaissance d'un objet sémantique dans ses relations avec d'autres objets sémantiques concernés par le champ de connaissance du mot.

À titre d'illustration, nous reprenons la représentation du noyau du mot travail, pratique humaine et valeur sociale épaisse, centrale dans la vie sociale ${ }^{16}$.



Schéma 1 : représentation du noyau du mot travail (d'après Galatanu, 2009c)

\subsubsection{Hypothèse 3}

La troisième hypothèse interne de la SPA avance l'existence d'un ensemble ouvert d'associations argumentatives des éléments du noyau avec d'autres représentations conceptuelles et sémantiques portées par des mots du lexique de la langue concernée, ancrées dans le cinétisme culturel de la communauté linguistique et, de ce fait, plus cinétiques. (Ibidem : 199) : l'ensemble ouvert des stéréotypes (Sts).

\subsubsection{Hypothèse 4}

31 La quatrième hypothèse avance ainsi que le noyau et l'ensemble ouvert d'associations stéréotypiques forment un dispositif génératif de sens discursif argumentatif potentiel. Ce processus de génération des Possibles Argumentatifs (PA) produit des séquences discursives virtuelles qui associent au mot même un élément de ses stéréotypes. Cet élément, compte tenu de la définition des stéréotypes, peut être un élément nucléaire, comme < effort > pour le mot travail, ou un élément extrinsèque au noyau, mais présent dans les stéréotypes, comme < souffrance > ou < fatigue ?, pour ce même mot. Ces deux derniers éléments sont justement associés à l'une des propriétés essentielles du mot travail, < effort `. Ainsi, parmi les PA générés par le dispositif N-Sts de travail, on peut trouver en français: travail DONC effort, travail DONC fatigue, travail DONC récompense/ salaire, travail DONC joie, etc. Comme les stéréotypes, ces séquences discursives potentielles participent d'un ensemble ouvert, dans lequel un locuteur francophone peut puiser les Déploiements discursifs Argumentatifs (DA) de sa signification, en mobilisant le mot travail dans ses actes de parole, en vertu de sa compétence sémantique ou plutôt sémantico-discursive. De même, un locuteur francophone peut reconnaître en vertu de cette compétence, des déploiements de la signification (DA) du mot travail habilités par les PA. Les PA font partie de la signification apprise et obligatoire du mot dans une communauté linguistique ${ }^{17}$.

32 Autrement dit, la signification d'une expression linguistique est faite de plusieurs niveaux de potentialités, fondées sur: (i) les associations de mots présentes dans le noyau - potentialités intrinsèques au noyau ; (ii) les éléments associés au noyau par les 
stéréotypes, extrinsèques au noyau, mais présents dans la signification à ce niveau, plus cinétique ; (iii) les possibles argumentatifs, id est les potentialités discursives du mot.

\subsubsection{Hypothèse 5}

33 La cinquième hypothèse est l'hypothèse centrale de la SPA, puisqu'il s'agit de l'interface des deux formes de manifestation simultanée du sens linguistique: la signification lexicale et le(s)sens discursif(s). Plus précisément, il s'agit des rapports entre les PA, déjà appréhendés par la configuration de la signification du mot et les déploiements discursifs - DA - de ce mot. C'est à l'interface des PA et des DA, de leurs rapports, que nous situons les mécanismes discursifs de génération du sens discursif et de (re)construction de la signification lexicale. C'est dans cet espace que se manifestent :

34 - La fonction du sens discursif dans la confirmation et la régénération des significations ou, au contraire, dans le vieillissement, voire la déconstruction - reconstruction des potentialités descriptives, argumentatives et axiologiques de celles-ci ; - Et, par voie de conséquence, le cinétisme des significations des mots. Cette hypothèse sous-tend le principe explicatif du fonctionnement du sens linguistique qui s'appuie sur deux contraintes qui agissent simultanément : contrainte de la signification sur la génération du sens discursif par déploiement du potentiel de cette signification (conforme ou non conforme au protocole sémantique du mot); contraintes du sens discursif sur l'interprétation d'une signification, confirmée ou régénérée, ou encore déconstruite et reconstruite dans et par l'activité de parole (Galatanu, $2018: 223$ ). Nous avons appelé ce phénomène «l'injonction paradoxale du sens linguistique $»$.

37 Comme on a pu le constater dans les exemples (1) à (3) ${ }^{18}$, la (re)construction de la signification par le sens discursif comporte en SPA une orientation axiologique, conforme au potentiel axiologique et donc argumentatif de la signification des mots, ou au contraire, une flexion de polarité.

Ce principe se veut, entre autres, une réponse au questionnement de Michel Bréal sur la tendance péjorative et la tendance " méliorative ", "d'affaiblissement » accompagné d'une sorte de "décoloration", "qui vient de ce que le mot est employé en toute espèce de groupements et d'associations». "[...] Celui qui s'en tient à l'étymologie sans prendre garde à l'affaiblissement des sens peut être amené à d'étranges erreurs [...]» (Bréal, [1897]1982: 105-106).

La flexion de polarité décrite par la SPA, très présente dans la zone sémantique qui nous intéresse ici, rejoint les remarques de Bréal sur un changement sémantique qui affecte, en diachronie, les pôles de l'orientation axiologique (cf. Bréal, Ibid : 102).

La SPA propose une approche de ces phénomènes susceptible d'expliquer le mécanisme sémantico-discursif de ces tendances. Les exemples donnés par Bréal peuvent avoir, selon les hypothèses de la SPA, leur origine dans le cinétisme sémantico-discursif. 


\section{Les mécanismes du cinétisme sémantico-discursif}

\subsection{Définition du concept et choix du terme}

41 Le concept de cinétisme discursif et sémantique renvoie à un double mouvement permanent: celui de l'impact du cinétisme culturel sur l'ensemble ouvert des stéréotypes rattachés aux propriétés essentielles du noyau et celui de l'impact du cinétisme linguistique sur le cinétisme culturel. Nous reprenons ici la dernière formulation de la définition du concept de cinétisme discursif et sémantique.

"Le cinétisme discursif de la signification lexicale est un "mouvement discursif" permanent de celle-ci dans l'environnement sémantique de la séquence de parole enrichissement ou appauvrissement, flexion de polarité, chargement ou déchargement de valeurs modales - le temps de l'occurrence de parole, mais pouvant se stabiliser et provoquer un cinétisme sémantique, voire même le changement sémantique. » (Galatanu, $2018: 18$ )

D'où le choix du mot cinétisme du grec kívnux, kínêma, mouvement ${ }^{19}$.

43 Le principe explicatif $d u$ fonctionnement $d u$ sens linguistique s'appuie sur trois caractéristiques de la signification lexicale : la complexité de la signification lexicale ${ }^{20}$, sa fluiditée ${ }^{21}$ et sa flexibilité2 2 .

\subsection{Quelques mécanismes sémantico-discursifs du cinétisme sémantique}

44 Dans ce qui suit, nous mentionnons et illustrons par des exemples tirés des travaux antérieurs dans ce domaine, quelques-uns des mécanismes sémantico-discursifs qui sous-tendent le processus de cinétisme sémantique. Les exemples choisis mobilisent des valeurs sociales complexes, épaisses, et donc des valeurs modales épaisses, plus sensibles au phénomène de cinétisme sémantique ${ }^{23}$.

Le déploiement discursif du potentiel - descriptif, argumentatif et orienté axiologiquement - de la signification des entités lexicales conforme (" conformiste ») au protocole sémantique du mot.

Nous rappelons qu'en SPA le protocole sémantique du mot permet de générer, à partir du dispositif formé du noyau et de l'ensemble ouvert des stéréotypes rattachés à ce noyau, des Possibles Argumentatifs, PA, qui sont des séquences discursives virtuelles, potentielles qui associent le mot même à l'un des éléments de ses stéréotypes. Ainsi, guerre a parmi ses stéréotypes et donc parmi ses PA le fait de tuer, de démolir, de détruire, PA qui sont déployés dans l'exemple 4.

(4) C'est une vraie guerre, elle tue, elle démolit, elle détruit.

Les flexions de polarité discursives (5) et sémantiques (6)

48

La flexion de polarité désigne une interversion discursive (exemple 5) ou sémantique (cinétisme sémantique proposée par l'instance discursive) des pôles axiologiques des valeurs qui participent à la signification lexicale (exemple 6). Dans ce dernier exemple, la réception de l'énoncé comme un énoncé sémantiquement acceptable s'appuie sur l'acceptation d'une propriété inédite de démocratie, construite discursivement, celle de l'inefficacité.

(5) C'est une sale guerre, mais elle va assurer l'indépendance de notre pays.

(6) C'est une démocratie, mais elle est efficace. 


\section{stéréotypes du mot, bloquant ainsi le déploiement des stéréotypes axiologiquement} positifs, qui distinguaient l'indignation de la colère.

\section{De la réclame à la publicité : un vieillissement sémantique et un changement de désignation}

Nous n'essayons pas ici de retracer l'histoire de la réclame et de la publicité, ni d'ailleurs celle des mots qui désignent à la fois une pratique sociale, une institution et des valeurs sociales épaisses. Notre objectif est de montrer le rôle du cinétisme discursif et sémantique dans la (re)définition d'une pratique en tant qu'institution sociale au sens de Searle (2010), par la reconstruction discursive du statut-fonctions des faits institutionnels qui la constituent, et par voie de conséquence, dans le vieillissement du mot réclame. Le changement de désignation de cette institution et de son ensemble des faits institutionnels apparaît comme une conséquence du vieillissement de ce mot. 

pratique (qui comporte, comme toutes les pratiques humaines et sociales, des valeurs modales épaisses), à travers les noyaux de réclame et de publicité. Nous nous appuyons principalement sur les définitions de plusieurs dictionnaires de la langue française ${ }^{25}$. Notons également que les dictionnaires consultés comportent la mention "vieilli » pour le mot réclame, et par ailleurs, indiquent comme dates d'usage installé dans la langue française de ces mots en tant que désignations des pratiques concernées : 1829 pour publicité et 1834 pour réclame. «La transition» de la réclame vers la publicité est datée des années 1930 et l'explication de cette transition proposée par les textes des spécialistes de son histoire ${ }^{26}$ est le fait que «la publicité " réfère à une pratique s'appuyant sur une « discipline » plus rigoureuse, plus organisée et conceptualisée, plus technique sinon scientifique, déclinant les principes de la communication de masse dans le domaine du commerce et de la propagande, de la promotion des projets sociétaux, etc.

Nous avons toutefois conforté cette analyse avec des textes institutionnels qui ont posé le statut-fonction de ces pratiques. Et nous adoptons également une approche introspective pour valider le caractère de propriété essentielle de chaque élément signifiant, avec le test : “ *ette pratique est parfois $x=$ élément signifiant nucléaire ${ }^{27}$. Ce test concerne la présence nécessaire des propriétés nucléaires pour la reconnaissance du mot, et donc de l'objet sémantique, appris et partagé par la communauté linguistique et culturelle. Dire que * La volonté d'assurer le succès de l'objet de la réclame est parfois l'objectif de la réclame paraît ainsi peu acceptable, tout comme l'énoncé *Le lapin a parfois quatre pattes est peu acceptable, voire pas du tout. Notons toutefois que le test sur les propriétés nucléaires des mots appartenant au champ sémantique des catégories naturelles est plus fiable que celui concernant les propriétés essentielles des mots dans le champ sémantique des catégories culturelles, plus perméables au cinétisme culturel.

Dans les schémas qui suivent, $X$ est l'agent producteur (direct ou indirect) du message publicitaire, $\mathrm{D}=$ le destinataire de la réclame/publicité, $\mathrm{O}=$ l'objet à faire valoir et à faire connaître (marchandise, objet culturel, etc.), $\mathrm{M}=$ le message publicitaire, DONC = le connecteur argumentatif abstrait qui assure l'organisation argumentative et vectorielle de la configuration de mots du noyau, $\mathrm{ET}=$ le connecteur coordonnant pour introduire des éléments ayant le même niveau dans la configuration vectorielle ${ }^{28}$.

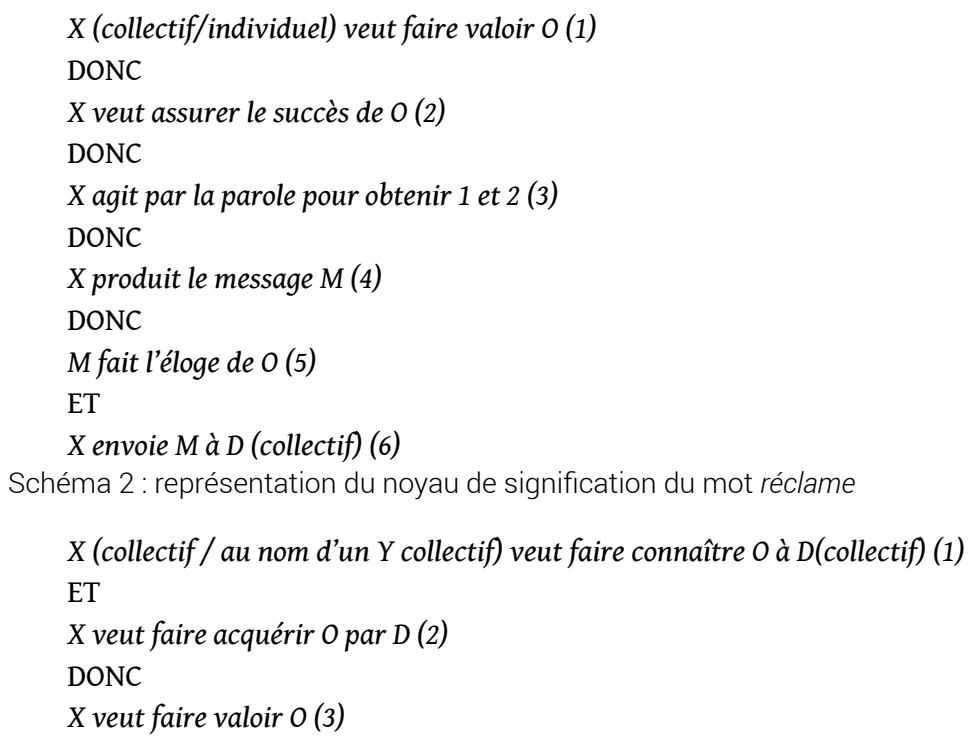


ET

$X$ veut agir par le discours (4)

DONC

$X$ produit le message $M(5)$

DONC

$M$ fait connaître $O$ (6)

ET

$M$ valorise (fait valoir) $O$ (7)

DONC

$X$ diffuse $M$ pour $D(8)$

Schéma 3 : représentation du noyau de signification du mot publicité

60

La comparaison des deux noyaux fait apparaître quelques différences entre les deux mots, dès ce niveau plus stable.

Ainsi, dans la configuration vectorielle d'éléments signifiants (mots, donc représentations sémantiques de ces mots) de réclame, <le faire valoir>, porteur de valeurs axiologiques très positives: esthétiques, pragmatiques, intellectuelles, voire même morales/éthiques occupe une position supérieure sur-modalisant un objectif d'ordre pragmatique, <assurer le succès> de l'objet dont l'éloge sera le contenu du message adressé aux destinataires. Dans le cas de publicité, l'élément qui occupe la position supérieure est la volonté de <faire connaître>, de rendre public un objet, de le proposer à l'acquisition et le <faire valoir> est la suite logique dans l'argumentation en faveur de cette acquisition.

tre recherche exploratoire, nous avons examiné des énoncés tirés de la base de données FRANTEXT qui mobilisent les deux mots à partir des dates mentionnées pour le début de leur usage en tant que dénominations de la pratique de promotion des objets, des idées et programmes politiques, etc.

avons ainsi identifié dans les textes de FRANTEXT 2603 occurrences de publicité entre 1828 et 2018 (année où s'arrête notre corpus) et 257 occurrences de la réclame ${ }^{29}$ entre 1843 et 2019 (année où s'arrête le corpus d'énoncés qui mobilisent cet item). Nous avons analysé l'environnement sémantique de ces mots, pour faire apparaître les déploiements discursifs de leurs significations, DA à orientation axiologique.

Dans l'espace de cet article, nous allons nous focaliser sur les énoncés qui mobilisent le mot réclame qui, selon nos hypothèses, subit discursivement parlant un double processus : d'axiologisation négative et de vieillissement. Le vieillissement a conduit ainsi à un changement de désignation dans la pratique de la publicité et dans les discours sur cette pratique. Le cinétisme de la pratique est un véritable processus d'amélioration sur le plan déontologique, de la technique et de l'efficacité commerciale.

Le tableau ci-dessous synthétise les résultats concernant le corpus d'énoncés comprenant la réclame. La source des catégories représentées dans le tableau est celle du noyau de signification du mot réclame (schéma 2) : l'action, c'est-à-dire le processus de production du message publicitaire, le message produit, résultat de ce processus. Une première étude du matériel empirique a montré que la bivalence sémantique sur le plan axiologique peut être préservé par le processus discursif. 
Tableau 1 : Le cinétisme discursif et sémantique de la réclame (axiologisation positive /négative) : total 257 occurrences

\begin{tabular}{|c|c|c|c|c|}
\hline Axiologisation & Positive & Négative & Bivalente & Total \\
\hline \multicolumn{5}{|l|}{ Désignation } \\
\hline $\begin{array}{l}\text { L'action } \\
\text { (le processus) }\end{array}$ & $\begin{array}{l}31 \text { occurrences } \\
(16,4 \%)\end{array}$ & $\begin{array}{l}138 \text { occurrences } \\
(73 \%)\end{array}$ & $\begin{array}{l}20 \quad \text { occurrences } \\
(10,6 \%)\end{array}$ & $\begin{array}{l}189 \text { occurrences } \\
(73,5 \%)\end{array}$ \\
\hline $\begin{array}{l}\text { Le message } \\
\text { (résultat } \mathrm{du} \\
\text { processus) }\end{array}$ & $\begin{array}{l}6 \quad \text { occurrences } \\
(10 \%)\end{array}$ & $\begin{array}{ll}33 & \text { occurrences } \\
(55 \%) & \end{array}$ & $\begin{array}{l}21 \text { occurrences } \\
(35 \%)\end{array}$ & $\begin{array}{l}60 \quad \text { occurrences } \\
(23,3 \%)\end{array}$ \\
\hline $\begin{array}{l}\text { Processus et } \\
\text { résultat }\end{array}$ & $\begin{array}{l}0 \text { occurrence } \\
(0 \%)\end{array}$ & $\begin{array}{l}4 \text { occurrences } \\
(50 \%)\end{array}$ & $\begin{array}{l}4 \quad \text { occurrences } \\
(50 \%)\end{array}$ & $\begin{array}{l}8 \text { occurrences } \\
(3,10 \%)\end{array}$ \\
\hline Total & $37(14,4 \%)$ & $\begin{array}{l}175 \text { occurrences } \\
(68,10 \%)\end{array}$ & $\begin{array}{l}45 \quad \text { occurrences } \\
(17,5 \%)\end{array}$ & 257 occurrences \\
\hline
\end{tabular}

Le tableau fait apparaître une polarisation majoritairement négative des valeurs attribuées à la pratique de la réclame (73\%) et une polarisation négative $(55 \%)$ du message produit dans le cadre de cette pratique nettement supérieure au déploiement positif $\mathrm{du}$ mot (10\%). Le déploiement neutre (que nous avons appelé bivalent) du message (35\%) est également inférieur à son déploiement négatif (55\%), mais supérieur à son déploiement positif (10,6\%). L'espace de cet article ne nous permet pas d'illustrer tous les cas présentés par ce tableau. Nous allons illustrer seulement les mécanismes sémantico-discursifs en œuvre dans l'axiologisation discursive et sémantique négative du mot réclame.

Une première hypothèse interprétative se dégage de ces pourcentages. Le processus d'axiologisation discursive négative est porté davantage par la pratique (individuelle ou collective) de la réclame que par le message produit dans cette pratique Nous allons limiter l'illustration de notre analyse au cinétisme aux occurrences de réclame-action (pratique, processus), puisque le noyau de signification de ce mot inclut dans sa configuration argumentative et vectorielle la production et l'envoi du message.

Parmi les mécanismes sémantico-discursifs du cinétisme du mot, la stéréophagie est la plus représentée dans notre corpus. Plus précisément, le déploiement des stéréotypes associés à l'un des éléments nucléaires, dans un enchaînement argumentatif, conduit au blocage des autres éléments nucléaires et de leurs stéréotypes et, d'autre part, à une flexion de polarité négative du mot. Ainsi, le pouvoir de la réclame d'assurer le succès d'un objet la transforme en une pratique totalitaire, qui « régente » tout, qui « domine nos vies".

(9) [...] C'est qu'entre-temps, telle une pieuvre $e^{30}$, la réclame s'est mise à tout régenter ; cette activité qui avait démarré comme une blague domine désormais nos vies : elle finance la télévision, dicte la presse écrite. (Beigbeder, F., 99 francs, 2000, p. 49) ${ }^{31}$

Par le mécanisme de stéréophagie déployant l'effet de réussite visé par cette pratique (assurer le succès de l'objet de la réclame), cet effet devient si fort, si puissant, qu'il règne et gouverne tout. 
(10) [...] cette franc-maçonnerie de la réclame règne et gouverne et défend la place à tout homme bien né: "c'est un menteur!» et avec ce mot-là, on le tue. (Goncourt Edmond de, Goncourt Jules de, Journal: mémoires de la vie littéraire, t.1: 1851-1863, mais 1856, Mai 1856, p. 234)

70 En tant que l'un des moyens les plus importants pour assurer le succès, la réclame, comme les concours, « surmène, étrique, surexcite et gâte » les hommes et en fait « des charlatans », « des intrigants $»^{32}$.

(11) [...] Les deux moyens pour y parvenir [au succès, NN), le concours et la réclame aboutissent à des effets du même genre. Ils surmènent, étriquent, surexcitent et gâtent l'homme. Le concours fait des éreintés et des bêtes de somme, la réclame fait des charlatans et des intrigants. [...] (Taine, H., Notes sur Paris : Vie et opinions de M. Frédéric Thomas Graindorge... recueillies et publiées par H. Taine, son exécuteur testamentaire, 1867, p. 298)

71 Un autre mécanisme très présent est celui de la flexion de polarité (orientant la réclame vers le pôle négatif des valeurs modales que ce mot porte), mécanisme basé sur la complexité modale du mot. Ainsi, le succès (valeur modale pragmatique positive) est assuré par un langage de lieux communs (valeurs modales intellectuelle et esthétique négatives), ou par «un usage du langage sans scrupule» (valeur morale négative ${ }^{\circ}$. L'exemple 12 illustre ce mécanisme.

(12) [...] et du commerce, qui en fait usage sans mesure ni scrupule. C'est ainsi qu'en un temps relativement court le vocabulaire de la métaphysique ${ }^{33}$ va alimenter le langage de la réclame. (Bréal, M., Essai de sémantique, 1897, ch. IX, Les prétendues tendances des mots : p. 105)

Les objectifs de la réclame (faire l'éloge d'un objet et lui assurer ainsi le succès par rapport aux objets concurrents) sont déployés aussi dans des énoncés qui proposent une flexion de polarité : disproportion entre l'objet et «sa réclame», message convaincant mais mensonger dangereux.

(13) [...] N'est-ce pas là pour les pauvres parents une charge bien grosse, et n'est-il pas affligeant de voir la disproportion entre la réclame et les produits? (Taine, H., Notes sur Paris..., 1867, p. 164)

Enfin, nous avons identifié un mécanisme de " contamination discursive " par les collocations du mot, plus précisément, par l'insertion de réclame dans l'énumération d'autres pratiques et valeurs sociales porteuses de valeur(s) négative(s), ou dans des syntagmes porteurs de valeurs affectives négatives.

(14) [...] Ces gens-là combattent pour établir le règne de l'évangile. Nous intéresser à mille choses qui touchent aux grands soucis de l'heure sans verser dans la banalité, la réclame, la politique, le charlatanisme. [...] (Barrès, M., Mes Cahiers, t. 11, juillet 1944février 1917, p. 136)

Les exemples du corpus qui réfèrent au passage de la réclame à la publicité montrent la charge discursive négative du premier et les bénéfices en termes de charge discursive positive du second. Cette période (1930-1931, selon les dictionnaires de la langue française ${ }^{34}$ ) et la période qui suit et qui va jusqu'en 2018 dans notre corpus, c'est la période où la publicité s'est imposée et le vieillissement de la réclame s'est confirmé. À titre d'illustration, dans l'exemple 15, qui est tiré d'un discours expert sur le commerce $^{35}$ et où les deux mots coexistent, l'auteur propose comme indicateur de la transition le passage du besoin individuel d'être informé et bien informé, à un besoin collectif de l'information. Nous rappelons toutefois qu'il s'agit ici d'une étude linguistique du cinétisme sémantique du mot réclame et de son remplacement par le mot publicité et non de l'analyse des pratiques publicitaires, de leur historique et de leur 
évolution, analyse qui relève du ressort des sciences de la communication publicitaire. Nous pensons que le point de vue observationnel linguistique peut éclairer le changement sémantique, certes lié aux changements de la pratique, sans pour autant remplacer le point de vue du dernier domaine scientifique mentionné.

(15) [...] Cette publicité collective pousse à la consommation du riz, du roquefort, des bananes ou de la confiture, à l'achat de gants ou de livres...la réclame - a dit un technicien de la publicité - a cessé d'ête un besoin exclusivement individuel, pour devenir un besoin collectif. C'est la publicité. (Coston, G.\& Coston, H., l'A.B.C. du journalisme : cours élémentaire en 30 leçons, 1952, p. 145)

L'exemple (16) explicite les acquis de cette pratique à partir de «la résistance à la fausse information ou simplement à l'information dûment contraignante ", notamment en vue d'augmenter la demande. Les valeurs implicites de ce passage sont celles d'une déontologie de la pratique publicitaire qui se met en place.

(16) [...] Chacune des grandes unités de production se propose d'augmenter la demande dont elle bénéficie par la publicité et la réclame; la résistance à la fausse information ou simplement l'information dûment contraignante est liée à une politique intelligente pratiquée par les acheteurs. (Perroux, F., L'Economie du XXe siècle, 1964, p. 403)

L'exemple (17) insiste sur les acquis de la réclame devenue publicité, sur le plan esthétique.

(17) [...] la publicité entre au musée, on organise des expositions rétrospectives d'affiches, on distribue des prix d'excellence, on la vend en cartes postales. Fin de l'âge de la réclame, vive la communication créative, la pub louche sur l'art et le cinéma, elle se prend à rêver d'embrasser l'histoire. [...] (Lipovetsky, G., L'Empire de

l'éphémère : la mode et son destin dans les sociétés modernes, 1987, p. 218)

77 Tout se passe comme si les discours auxquels appartiennent ces énoncés, parlant de la réclame, en tant que pratique et que message construit dans et par cette pratique, avaient chargé sa désignation de valeurs négatives portant sur les objectifs, la technique de production des messages et leurs effets. Ces discours font apparaitre la discordance entre l'objet et la promotion de l'objet, entre les objectifs et les effets de la réalisation de ces objectifs. Et comme si le même mot ne pouvait plus porter aussi les améliorations souhaitées de cette pratique, le mot publicité, longtemps co-existant avec le mot réclame, a fini par « évincer » ce dernier.

Ceci nous amène à formuler une autre hypothèse interprétative locale sur les liens entre la pratique humaine et sociale de promotion et même d'auto-promotion par des messages publics et sa désignation. Nous pensons que ce n'est pas le manque de technicité et de savoir sur la communication qui se trouve à l'origine du vieillissement du mot réclame, mais le cinétisme discursif et sémantique, s'appuyant sur la critique des dérives et des lacunes de cette pratique, qui a conduit au changement de la pratique et de ses valeurs sociales épaisses, en instaurant sa professionnalisation et en construisant un sens linguistique positif du mot concurrent pour la désigner.

\section{Conclusion}

79 Notre étude exploratoire dans ce domaine conforte l'hypothèse interprétative locale et les hypothèses concernant le rôle du cinétisme discursif et sémantique dans le vieillissement d'un mot et dans le changement de désignation. Certes, notre corpus réduit ne nous permet pas encore de valider nos hypothèses, mais les confortent et 
permettent d'envisager une méthodologie de la recherche basée sur la triangulation de données discursives et expérimentales auprès des sujets parlants. Dans le cas de la réclame et de la désignation de la pratique de promotion commerciale, politique, idéologique qui s'impose, la publicité (et la pub, pour désigner le message), une étude des nombreuses occurrences de cette dernière nous permettra de suivre le processus du cinétisme discursif et sémantique et de flexion de polarité (d'axiologisation négative de certains éléments de cette pratique) que la nouvelle désignation subit à son tour. Notre hypothèse est que l'axiologisation négative $d u$ mot publicité va porter tout particulièrement sur sa présence intrusive et agressive dans les médias et sur les aspects financiers qui lient cette pratique aux différentes formes de production artistique dans ces médias.

Si le cinétisme de la signification lexicale, contraint par le sens discursif et agissant sur l'humain et le social, participe à sa (re)construction permanente, notamment dans la zone sémantique des valeurs sociales et humaines épaisses (y compris l'artefact appartenant au patrimoine culturel), qu'en est-t-il de la zone sémantique des catégories artéfactuelles et naturelles? Nous souhaitons conclure par cette question et par son corollaire: le cinétisme discursif peut-il entraîner une recatégorisation discursive fréquente, systématique, du sémantisme des mots qui désignent les objets de la publicité, comme dans les exemples suivants qui sont des énoncés publicitaires récents? Car dans ces exemples, la viande et le pain sont investis de propriétés qualifiant l'humain et non ce qu'il produit.

(18) Il a compris qu'il peut manger mieux grâce à une viande de qualité supérieure, responsable et durable. (Publicité télévisée pour LIDL)

(19) C'est du bon pain, moelleux et solidaire. (Publicité télévisée).

\section{BIBLIOGRAPHIE}

ADAM, J.-M., BONHOMME, M., 2012, L'argumentation publicitaire. Rhétorique de l'éloge et de la persuasion, Paris, Nathan Université.

BELLACHHAB, A., 2019, «Quand déclarer c'est faire identité. Vers une ontologie de l'identité discursive à travers des lettres de tueurs en série ", dans A. Steuckardt et K. Collette (éds.), Ecrits hors-normes, Sherbrooke, Éditions de l'Université de Sherbrooke, p. 115-126.

BERTHELOT GUIET, K., 2015, Analyser les discours publicitaires, Paris, Armand Colin.

BLANK, A., 2000, « Pour une approche cognitive du changement sémantique lexical : aspect sémasiologique ", dans J. François et alii, Théories contemporaines du changement sémantique. Mémoires de la Société de Linguistique de Paris, Tome IX, Leuven, Peeters, p. 59-74.

BONHOMME, M., ADAM, J.-M., 2000, Analyses du discours publicitaire, Toulouse, Éditions Universitaires du Sud.

BONHOMME, M. (dir.), 2013, Semen, 36, Les nouveaux discours publicitaires. https://doi.org/ 104000/semen.9599.

BRÉAL, M., 1897, Essai de sémantique. Sciences des significations, Paris, Hachette. 
CHAILLOU, M., 2011, Le phénomène d'axiologisation discursive de la signification lexicale : le cas de « harmonisation » dans le cadre de l'Union Européenne, Thèse de doctorat soutenue à

l'Université de Nantes.

CHARAUDEAU, P., 1994, «Le discours publicitaire, genre discursif », Revue Mscope, nº 8 . CRDP de Versailles. http://www.patrick-charaudeau.com/Le-discours-publicitaire-genre.html

CHUPIN, I., HUBÉ N., KACIAF, N., 2009, Histoire politique et économique des médias en France, Paris, La Découverte.

COZMA, A.-M. et GALATANU, O., 2019, « La construction discursive dévalorisante du concept de démocratie », Neuphilologische Mitteilungen, Bulletin de la Société Néophilologique, II CXIX 2018, Helsinki, p. 249-272.

DOTOLI, G., 2018, Le Dictionnaire historique de la langue française d'Alain Rey, Paris, Hermann.

FRANÇOIS, J. et alii, 2000, Théories contemporaines du changement sémantique. Mémoires de la Société de Linguistique de Paris, Tome IX, Leuven, Peeters.

FRANÇOIS, J. et GEERAERTS, D., 2016, « Pour une vision périphérique de la sémantique présente et à venir ", dans J. François et D. Geeraerts (dir.), Langages, 201. La sémantique linguistique en vision périphérique, p. 5-14.

GALATANU, O., 1999, « Le phénomène sémantico-discursif de déconstruction-reconstruction des topoï dans une sémantique argumentative intégrée », dans O. Galatanu et J.-M. Gouvard (dir.), Langue française, 123, p. 41-51.

GALATANU, O. (2000a). « Langue, discours et systèmes de valeurs », dans E. Suomela-Salmi (dir), Curiosités linguistiques, Turku, Presses Universitaires de Turku, p. 80-102.

GALATANU, O., [1998] 2000b, « La reconstruction du système de valeurs convoquées et évoquées dans le discours médiatique », dans A. Englebert, M. Pierrard, L. Rosier \& D. Van Raemdonck (dir.), Actes du XXIIe Congrès International de Linguistique et Philologie Romanes, Bruxelles, Tübingen, Max Niermeyer Verlag, p. 251-258.

GALATANU, O., 2002, "Le concept de modalité : les valeurs dans la langue et dans le discours ", dans O. Galatanu (dir.), Les valeurs, Nantes, MSH Ange Guépin, p. 17-32.

GALATANU, O., 2003, « La sémantique des possibles argumentatifs et ses enjeux pour l'analyse de discours ", dans M. J. Salinero Cascante et I. Iñarrea Las Heras (dir.), El texto como encrucijada: estudios franceses y francófonos, Vol.2, Lograno, p. 213-225. [disponible en ligne].

GALATANU, O., 2006, « Du cinétisme de la signification lexicale », dans J.-M. Barbier et M. Durand, Sujets, activités, environnements, Paris, Presses Universitaires de France, p. 85-104.

GALATANU, O., 2009a, " Semantic and discursive construction of the 'Europe of knowledge' ", dans E. Suomela-Salmi et F. Dervin (dir.), Cross-Linguistic and Cross-Cultural Perspectives on Academic Discourse, Amsterdam/Philadelphia, John Benjamins, p. 275-296.

GALATANU, O., 2009b, « La "stéréophagie", un phénomène discursif de déconstructionreconstruction de la signification lexicale », dans I. Evrard, M. Pierrard, L. Rosier et D. Van Raemdonck (dir.), Représentations du sens linguistique III. Actes du colloque international de Bruxelles (2005), Bruxelles, De Boeck/Duculot, p. 198-208.

GALATANU, O., 2009c, « Les incidences sémantiques des déploiements argumentatifs dépendants du co-(n)texte de production du discours », dans E. Havu, J. Härmä, M. Helkula, M. Larjavaara et U. Tuomarla (éds.), Mémoires de la Société Néophilologique de Helsinki. LXXVIII, La langue en contexte, Helsinki, University of Helsinki, p. 392-404. 
GALATANU, O., 2010, « Pour une approche sémantico-discursive du concept d'identité : faute, crime et dynamique discursive », dans M. Palander-Collin et alii (éds.), Mémoires de la Société Néophilologique de Helsinki. LXXXI. Constructing Identity in Interpersonal Communication, Helsinki, University of Helsinki, p.125-138.

GALATANU, O., 2018, La sémantique des possibles argumentatifs. Génération et (re)construction discursive du sens linguistique, Bruxelles-Bern-Berlin-New York-Oxford-Wien, P.I.E. Peter Lang.

GALATANU, O., 2019, « Du discours hors-normes d'un tueur en série à la reconstruction discursive de soi et de l'espace épistolaire : une normalité revendiquée et régénérée ", dans A. Steuckardt et K. Collette, Ecrits hors-normes, Sherbrooke, Editions de l'Université de Sherbrooke, p. 92-114.

GEERAERTS, D., 1991, « La grammaire cognitive et l'histoire de la sémantique lexicale », Communications, $\mathrm{n}^{\circ} 53$, p. 17-50.

GOSSELIN, L., 1997, « Les études psycholinguistiques sur la compréhension des expressions ambigües : Une critique linguistique », dans J. François et G. Denhière (dir.), Sémantique linguistique et psychologie cognitive. Aspects théoriques et expérimentaux, Grenoble, Presses Universitaires de Grenoble, p. 75-115.

GUILLAUME, G., [1929]1984, Théorie des aspects, des modes et des temps, suivi de L'architecture du temps dans les langues classiques, Paris, Honoré Champion.

GUYOT, J.,1992, L'écran publicitaire. Idéologie et savoir-faire professionnels de la publicité dans l'audio-visuel (1968-1976), Paris, L'Harmattan.

HERANIC, T., 2017, Le travail c'est la santé, mais la paresse ne tue pas : construction du sens discursif du lexique. Etude comparative de l'anglais américain et du français, Thèse de doctorat soutenue à l'Université de Nantes.

KLEIBER, G., 1984, « Dénomination et relations dénominatives », Langages, 76, p. 77-94.

KLEIBER, G., 2003, « Sur la sémantique de la dénomination », Verbum, 25(1), p.97-107.

KOCK, P., 2000, « Pour une approche cognitive du changement sémantique lexical : aspect onomasiologique ", dans J. François et alii (éds.), Théories contemporaines du changement sémantique. Mémoires de la Société de Linguistique de Paris, Tome IX, Leuven, Peeters, p.75-95.

LEBAS, F., 2017, « L'arnaque de l'étymon du mot travail ». réseau-salariat.info, mise en ligne le 11 mai 2017. Consulté le 19 janvier 2020.

LE NY, J.-F. et FRANQUART-DECLERCQ, C., 2001, « Flexibilité des significations : traits sémantiques et compréhension des métaphores verbales », Verbum, 23, p. 385-400.

LONGHI, J. et SARFATI, E.G.-E., 2014, « Les discours institutionnels. Contribution à l'analyse des discours institutionnels et politiques », Mots, 108, p. 167-170.

MARCUS-STEIFF, J., 1983, « Publicité », dans J. Bersani, H. Schweizer et alii (éds), Encyclopaedia Universalis, vol. 13, Paris, Encyclopædia Universalis France, p. 799.

MARTIN, R.,1990, « La définition naturelle ». dans J. Chaurand et F. Mazière (éds.), La définition, Paris, Larousse, p. 86-95

NIKOLENKO, V., 2015, Le cinétisme de la signification lexicale dans la zone sémantique de l'axiologique. Thèse de doctorat soutenue à l'Université de Nantes. 
OGER, C. et OLLIVIER-YANIV, C., 2003, « Conjuguer analyse du discours institutionnel et sociologie compréhensive ; vers une anthropologie du discours institutionnel », Mots, 71, p. 125-145.

PUTNAM, H., 1975, The Meaning of Meaning. Philosophical Papers. Vol. 2, Cambridge University Press.

PUTNAM, H., 2005, Ethics without Ontology, Cambridge (E.U.), Harvard University Press.

Rey, A. (dir.) ([1998]2019). Le Dictionnaire historique de la langue française, Paris, Le Robert (ISBN 2-85036-532-7).

SEARLE, J. R., 1969, Speech Acts, Cambridge, Cambridge University Press.

SEARLE, J.R., 1975, « A Taxonomy of Illocutionary Acts », dans Language, mind and knowledge. Studies of philosophy, Minnesota, Minneapolis, University of Minnesota Press, p. 344-369.

SEARLE, J. R., 2010, Making the social World, Oxford, Oxford University Press.

SOULAGES, J.-C. (dir.), 2015, L'analyse de discours. Sa place dans les sciences du langage et de la communication. Hommage à Patrick Charaudeau, Rennes, Presses Universitaires de Rennes.

SWEETSER, E., 1990, From Etymology to Pragmatics: Metaphorical and Cultural Aspects of Semantic Structure, Cambridge, Cambridge University Press.

\section{NOTES}

1. Se référer à Galatanu (1999, [1998] 2000b, 2006, 2018).

2. Pour la synthèse de ce point de vue, voir Galatanu (2018).

3. Ces limites sont imposées par « une zone de confort sémantique » des propriétés essentielles qui forment le noyau (cf. Galatanu, 2018 : chapitres 3 et 4).

4. Ces exemples sont construits, dans une démarche introspective visant à rendre plus clairs les mécanismes sémantico-discursifs du cinétisme de la signification lexicale. Pour une explication détaillée de cette démarche, voir François \& Geeraerts (2016), Galatanu (2018: 21-24). Nous signalons aussi une étude du cinétisme du mot démocratie, à partir d'un corpus recueilli sur le site de Paris Match se composant des discours des cinq principaux candidats aux élections présidentielles de 2017 (Cozma \& Galatanu, 2019).

5. Pour le rôle des actes déclaratifs dans la construction du monde social, voir Searle (2010).

6. Cette hypothèse sur l'impact du discours et de la reconstruction des significations lexicales que le discours propose ouvre, bien sûr, la voie à des recherches quantitatives sur des corpus importants dont le protocole est à construire.

7. Nous avons étudié ailleurs (Galatanu, 2009b : 200-203) le cinétisme discursif et sémantique du mot innovation et son changement sémantique dans une perspective sémasiologique. Ce changement est marqué par une "énucléation » : l'élément signifiant "désir de changement » disparaît pratiquement du noyau, en faveur de «la volonté de changer » et il s'inscrit comme élément possible dans l'ensemble ouvert des stéréotypes. Par ailleurs, l'insertion dans cet ensemble d'éléments stéréotypiques du <bénéfice collectif> de l'innovation participe à un phénomène de flexion de polarité. L'innovation perd ainsi sa valeur pragmatique négative. L'exemple cité par Littré "Ce n'est qu'une innovation " devient improbable, alors que "C'est une vraie innovation", devient un véritable éloge de l'action humaine, éloge décliné sous de nombreuses formes. L'acte d'innover devient une véritable valeur sociale complexe (que nous allons appeler valeur sociale complexe, épaisse, de par l'épaisseur de sa modalisation interne, comme nous allons le voir dans ce qui suit). 
8. Par une définition conventionnelle a posteriori (Martin, 1990).

9. Pour la modalisation interne à la signification des mots comme démocratie, crime, délit, justice, etc., voir, entre autres, Galatanu (2000, 2002, 2009a, 2010, 2018, 2019), Chaillou (2011), Nikolenko (2015), Bellachhab (2019), Cozma \& Galatanu (2019).

10. Voir à titre d'illustration de ces débats : Rey, A. (dir.), Le Dictionnaire historique de la langue française, Le Robert ([1998] 2019), (ISBN 2-85036-532-7), Dotoli (2018), Lebas, «L'arnaque de l'étymon du mot travail » [archive], réseau-salariat.info, 11 mai 2017, consulté le 19 janvier 2020.

11. Searle parle de fonction-statut, marquant ainsi le fait que l'attribution d'une fonction fonde un fait institutionnel. Nous inversons les termes et parlons, tout au moins dans la cadre de cette recherche, de statut-fonctions, pour mieux faire apparaître le fait qu'un statut de fait institutionnel peut s'appuyer sur une pluralité de fonctions.

12. Pour une présentation détaillée des sources de réflexion qui ont conduit à l'élaboration de la SPA, de ses postulats et hypothèses et de l'ensemble des concepts mis en œuvre pour la construction d'un modèle du fonctionnement sémantique, voir Galatanu (2018).

13. Nous reprenons, dans l'explicitation de la SPA, une partie des critères posés par Geeraerts (1991) pour présenter les grands courants de pensée en sémantique.

14. Ces propriétés sont organisées dans une configuration où leurs places ne peuvent pas être interchangées sans changer l'identité sémantique du mot : par exemple, pour le mot soldat, on ne peut pas intervertir les éléments du noyau du mot soldat « devoir combattre donc avoir un fusil donc pouvoir tuer » en " pouvoir tuer donc devoir combattre donc avoir un fusil » (exemple tiré de la thèse de doctorat de Bourmalo (2007). Pour l'explication détaillée de la configuration vectorielle du noyau, se référer à (Galatanu, 2018 : 163-165)

15. Nous adhérons à l'idée du philosophe Putnam (1975) sur la partie stable de la signification d'un mot, apprise et partagée par une communauté linguistique, le noyau, tout en proposant une autre configuration de cette partie. Il ne s'agit plus de traits nécessaires et suffisants pour reconnaître un objet du monde désigné par un mot, mais d'autres mots de la langue d'appartenance de ce mot, des propriétés essentielles pour sa reconnaissance, id est pour la reconnaissance d'un objet sémantique construit par les inter-discours de la communauté linguistique et culturelle. De ce fait, la catégorisation que le noyau propose relève d'une ontologie sémasiologique, sémantique et non d'une ontologie onomasiologique proposée par la philosophie et/ou les sciences sociales, ou même les sciences de la vie et de la terre. Pour une discussion détaillée et argumentée des spécificités du noyau de signification en SPA, voir (Galatanu, 2018 : 187-198).

16. Nous renvoyons le lecteur à nos travaux résumés dans Galatanu (2018) et à la thèse de doctorat de Tracy Heranic (2017), sur les mots travail et work.

17. L'étude du mot travail que nous résumons ici s'appuie sur l'analyse de plusieurs corpus, dont nous citons les textes authentiques et construits de trois manuels de français langue étrangère pour avancés (Galatanu, 2009c) et celui recueilli par Heranic (2017) auprès de 120 jeunes locuteurs de langue française et d'anglais américain à partir d'un protocole habilité par la sémantique des possibles argumentatifs. Nous renvoyons le lecteur à la présentation détaillée de sa méthodologie de recueil et de traitement de données.

18. Nous renvoyons le lecteur à la bibliographie de l'ouvrage qui présente et argumente la SPA (Galatanu, 2018). Nous précisons que dans nos analyses, nous utilisons une méthodologie basée sur la triangulation de plusieurs sources : les illustrations des définitions dictionnairiques, par des exemples attestés ou construits par le lexicographe ; des données issues d'enquêtes menées auprès de différentes catégories socioculturelles (au sens large du terme culturel, intégrant les cultures professionnelles) de locuteurs ; des données issues de l'analyse, de plus en plus outillée, de corpus discursifs. Cette méthodologie a été investie dans la présente recherche sur réclame et publicité (voir la section 4 de cet article). 
19. Nous précisons que le choix de ce terme, pour désigner «l'ensemble (ouvert) des variations discursives de la signification des expressions linguistiques, notamment des entités lexicales », se justifie par la nature de ces variations : changement de valeurs axiologiques, par une flexion de polarité discursive, insertion de nouveaux éléments, ne serait-ce que le temps de l'occurrence de parole, etc. Le rapprochement avec le concept de cinétisme en psychomécanique (G. Guillaume, [1929]1984) s'inscrit dans ce que nous avons décrit comme des résonances du modèle SPA. Cette résonance avec la psychomécanique s'appuie sur l'idée que la langue est toujours en train d'être construite, et lors de chaque occurrence de parole, saisie à un moment donné de sa construction. Des différences importantes, liées au domaine empirique observé et au point de vue observationnel du langage, justifient par ailleurs le fait que la SPA ne s'inscrit pas dans la filiation de la psychomécanique.

20. La complexité de la signification lexicale est due à la fois à son hétérogénéité (statut différent des différentes strates, degré différent de stabilité et de saillance des associations argumentatives) et à la présence dans sa configuration de représentations sémantiques d'autres mots, aussi complexes au niveau sémantique.

21. Nous définissons la fluidité de la signification lexicale comme "son potentiel d'évocation et de mobilisation d'autres significations de mots du lexique de la langue, qu'il s'agisse (a) de mots qui participent à sa configuration d'associations argumentatives [...], ou (b) de mots qui la "contaminent" dans l'environnement sémantique du sens discursif [...], ou même d'une contamination par son environnement contextuel » $(2018: 182)$.

22. La flexibilité sémantique se manifeste « sous la forme d'une variabilité des significations d'un locuteur à l'autre et pour le même locuteur » (Le Ny \& Franquart-Declercq, 2001). Elle est pour Gosselin (1997) une sorte d'indétermination. En SPA, cette variabilité va jusqu'à la déconstruction-reconstruction de la signification lexicale.

23. Nous ne pouvons pas décrire en détail ces mécanismes dans l'espace de cet article. Nous renvoyons à l'ouvrage de 2018 (Galatanu, 2018 : 231-255).

24. Il s'agit des articles de Marcel Conche, Jean-Michel Besnier et Laurent Maquet publiés le 13 octobre 2001 dans «Indignations ». Pour les détails de l'analyse, voir Galatanu (2003) et surtout Galatanu (2018 : 246-247).

25. Le Grand Robert, 2001 ; le Petit Robert, 2000, le Littré, [1877] 2003, TLFi: Trésor de la langue Française informatisé, http://www.atilf.fr/tlfi, ATILF-CNRS \& Université de Lorraine.

26. À titre d'exemples: Marcus-Steiff (1983), Chupin (Hubé et Kaciaf, 2009), Guyot (1992). Mentionnons également qu'il existe de nombreux travaux d'analyse linguistique des discours publicitaires : par exemple, Adam et Bonhomme (2012), Guiet (2015), Bonhomme (dir.) (2013), Bonhomme et Adam (2000), Charaudeau (1994), Soulages (dir.) (2015).

27. Cf. Galatanu (2018).

28. Pour une explicitation de la (re)présentation de la représentation sémantique des mots, se référer à Galatanu (2018: 282-289). Nous rappelons ici seulement que les propriétés proposées par les définitions des dictionnaires explicatifs du français sont testées comme propriétés essentielles à la reconnaissance du mot.

29. L'espace de l'article ne nous permet pas d'analyser l'ensemble du corpus obtenu avec les entrées : la réclame, une réclame, des réclames, sans réclame, avec réclame, que nous avons saisies pour distinguer le nominal des entrées du verbe réclamer.

30. C'est nous qui soulignons par des italiques.

31. Nous rappelons que tous les exemples qui suivent sont tirés du corpus construit à partir de FRANTEXT et que, visant à illustrer les mécanismes sémantico-discursifs d'axiologisation négative, ils ne sont pas organisés chronologiquement dans cette partie de l'exposé.

32. Signalons toutefois que dans l'exemple qui suit, il s'agit précisément de la promotion, des artistes par eux-mêmes ou de leurs discours. 
33. Notons que les analyses sont unanimes à marquer aujourd'hui l'usage d'un langage (para)scientifique dans les publicités pour les produits cosmétiques, allant jusqu'à l'emploi de la formule "prouvé scientifiquement».

34. Le Petit Robert, 2000 et Le Robert, 2001.

35. L'énoncé n'est pas marqué dans la base de données comme appartenant à un discours expert, nous le mentionnons en nous appuyant sur le titre du texte.

\section{RÉSUMÉS}

L'objectif premier de cet article est de proposer, argumenter et illustrer une approche complémentaire de celles qui étudient le changement sémantique et deux des phénomènes qui lui sont associés : le vieillissement et l'innovation dans le domaine lexical. Le corollaire de cet objectif est de bâtir un pont conceptuel et opérationnel entre le principe explicatif du sens linguistique proposé dans le cadre de la sémantique des possibles argumentatifs - le cinétisme discursif et sémantique - et les fonctions du langage dans les changements du monde social. L'illustration de cette proposition théorique mobilise un domaine social et linguistique particulièrement sensible : celui des valeurs sociales complexes et ipso facto des valeurs modales épaisses. L'analyse porte sur les mots réclame et publicité et sur les pratiques sociales qu'ils désignent.

The primary objective of this article is to propose, argue and illustrate an approach that is complementary to those that study semantic change and two of the phenomena associated with it: ageing and innovation in the lexical field. The corollary of this objective is to build a conceptual and operational bridge between the explanatory principle of linguistic meaning proposed within the framework of the semantics of argumentative possibilities - discursive and semantic kinesis - and the functions of language in changes in the social world. The illustration of this theoretical proposal mobilizes a particularly sensitive social and linguistic domain: that of complex social values and ipso facto thick modal values. The analysis focuses on the French words réclame (advertisement) and publicité (publicity) and on the social practices they designate.

\section{INDEX}

Mots-clés : cinétisme discursif et sémantique ; sémantique des possibles argumentatifs ; valeurs sociales et modales épaisses ; vieillissement sémantique ; changement de désignation.

Keywords : discursive and semantic kinesis; semantics of argumentative possibilities; thick social and modal values; semantic ageing; change of designation.

\section{AUTEUR}

\section{OLGA GALATANU}

Université de Nantes, groupe de recherche sur la construction discursive des représentations linguistiques et culturelles - CoDiRe \& EA 7469 PREFics, UBS 